\title{
A Thin-Film Infrared Absorber using CNT/Nanodiamond Nanocomposite
}

\author{
Yu Sui ${ }^{1}$, Vikrant J. Gokhale ${ }^{1}$, Olga A. Sherendova ${ }^{2}$, Gary E. McGuire ${ }^{2}$, and Mina Rais-Zadeh ${ }^{1}$ \\ ${ }^{1}$ Department of Electrical Engineering and Computer Science, University of Michigan, Ann \\ Arbor, MI 48105, USA \\ ${ }^{2}$ International Technology Center, Raleigh, NC, 27617, USA
}

\begin{abstract}
This paper reports on the fabrication and characterization of thin-film nanocomposites comprised of tangled carbon nanotubes in a polymer matrix. The density of nanotubes in the polymer was significantly increased using detonation nanodiamonds. Nanodiamonds reduce the surface forces between the polymer and the nanotubes and mitigate the agglomeration problem of nanotubes in polymer. This resulted in thinner, more uniform networks that serve as efficient absorbers of infrared energy over a broad spectrum, ranging from the visible to the midwavelength infrared. An infrared absorbance of $97 \%$ was achieved for a $1.6 \mu \mathrm{m}$ thick nanocomposite film across the spectral range of $714 \mathrm{~nm}$ to $5 \mu \mathrm{m}$. The films are electrically insulating, mechanically and thermally stable up to $300{ }^{\circ} \mathrm{C}$, and can be integrated with microbolometers to enhance their responsivity.
\end{abstract}

\section{INTRODUCTION}

Broadband infrared (IR) detectors enable night vision, imaging, and non-destructive testing of objects and thus can be used in medical diagnosis, industry, space, and defense applications. Infrared detectors are either uncooled or cooled. Generally, uncooled (or thermal) detectors offer a much wider bandwidth and work at higher temperatures as compared to cooled IR detectors [1]. The fundamental working principle of thermal detectors is that the absorption of incident radiation as heat causes a physical change in the sensor, such as resistivity change or frequency shift. This physical change is transduced into an electrical signal which is proportional to the radiated IR power. The IR absorption efficiency and thereby the sensitivity of thermal detectors can be improved by the addition of a thin-film IR absorber.

This paper presents carbon nanotube (CNT) based polymer composites that exhibit high IR absorptivity even at thicknesses less than $2 \mu \mathrm{m}$. Charged detonation nanodiamond particles (DND), produced from carbon-containing explosives are used to enhance the dispersion of CNTs in the polymer matrix. The CNT/DND polymer films are electrically insulating, mechanically robust, and thermally stable, making them ideal candidates for thin-film infrared absorbers.

\section{THEORY}

Various approaches have been taken for implementing IR absorbers. These include metallic thin films [2], quarter wavelength resonant cavities [3], metal blacks [4]-[6], carbon or graphite blacks [7], [8], and nanostructured metamaterials [9]. While good IR absorption characteristics have been obtained following these approaches, the reported IR absorbers are either temperature 
unstable, have a narrow absorption spectrum, or need to be deposited in a thick film to achieve high absorption. Specifically, quarter-wavelength cavities are too thick and wavelength specific. Metal alloy films, porous metals, and metal blacks have issues with aging and film stability [10]. Amongst different IR absorbers, those based on CNTs have shown the most promising results in terms of absorption efficiency (i.e., absorptivity per unit thickness). For example, CNT-based IR absorber paints with absorbance of $90 \%$ have been demonstrated [10]. In a different approach, up to $98 \%$ absorbance was reported for a $460 \mu \mathrm{m}$ thick vertically aligned forest of CNTs [11]. In both cases, one conclusion that is drawn is that while an individual CNT has similar absorbance characteristics to elemental carbon, the unique low-density and sparse network formed by aligned or disordered CNTs is the key factor in imparting the surface with radiation trapping characteristics approaching that of an ideal black body. A thick forest of aligned nanotubes is incompatible for use with low-profile thin-film bolometers but the concept of trapping photons using the unique one-dimensional structure of CNT forests can be extended to a random porous network of horizontally dispersed CNTs (figure 1). Such a network can be realized by spin or spray coating the nanotubes onto a substrate. To ensure mechanical stability of the film, a polymer can be used as the matrix material. However, the problem with mixing the CNT in a polymer solution has been the agglomeration of CNTs in the polymer when the density of CNT passes a certain threshold (typically $<\sim 0.01 \mathrm{wt} . \%$ ), which results in a non-uniform and thus low IR absorbance film. This work reports, for the first time, on thin and uniform CNT-based polymer films with exceptionally high IR absorptivity.
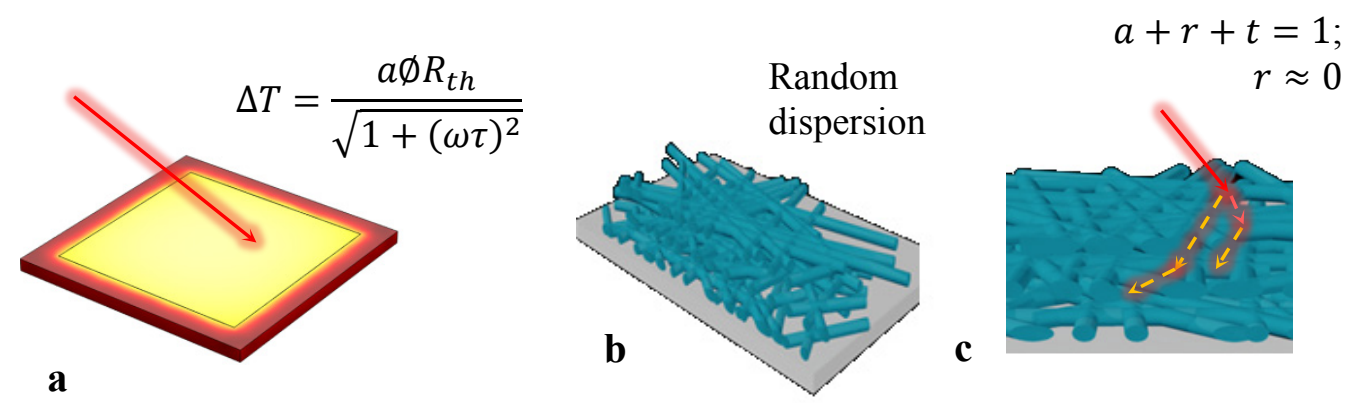

Figure 1. (a) Temperature change of the IR detector surface is dependent on the IR absorption coefficient, $a$. (b) Conceptual illustration of a dispersed random network of CNTs embedded in a polymer matrix. (c) The porous structure traps IR photons and absorbs them efficiently over multiple reflections inside the network.

\section{EXPERIMENTAL DETAILS}

\section{Fabrication of CNT nanocomposites}

Different approaches were taken in this work to obtain thin $(<2 \mu \mathrm{m})$ CNT-based IR absorbers. In the first approach, multi-walled nanotubes (MWNTs) suspensions were electrosprayed by Nano-Lab Inc. [12] onto a substrate. Figure 2 shows images of glass substrates coated with ultra-thin layers of CNTs. Although high IR absorptivities were achieved, as will be shown later, the electrosprayed MWNTs were only held on to the substrate by weak surface forces, and could be easily removed or scratched off from the surface. This complicates 
further processing and patterning of the films. Hence, a polymer is critical to ensure the mechanical stability and strength of the film.

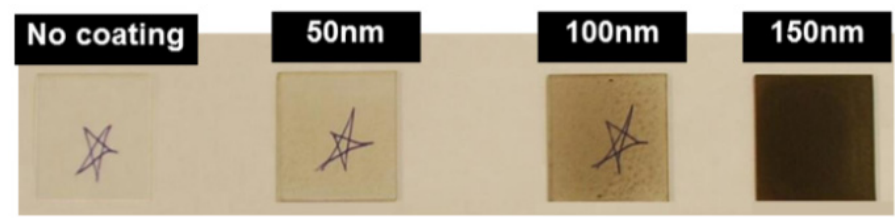

Figure 2. Electrosprayed MWNT films with different thicknesses on glass slides.

In the second approach, carbon nanotubes were dispersed in a polymer matrix using a dispersant. Various combinations of MWNT powder acquired from Cheap Tubes Inc. [13] along with poly-(methylmethacrylate) (PMMA, MicroChem Corp. [14]) as the polymer, and dimethylformamide (DMF, Fisher Scientific [15]) as the dispersant were used in initial experiments. MWNT powder was added to the required amount of DMF, mixed with uncured low-viscosity PMMA, and sonicated. Ultrasonication has been used for making nanocomposites with low-density CNTs in a polymer [16]. Most successful techniques rely on casting to form films with thicknesses on the order of tens of micrometers. In this case, the requirements for uniform films on the order of $2 \mu \mathrm{m}$ thickness necessitated spinning, which is a cheap and scalable deposition method. However, there was a significant amount of agglomeration (figure 3 ), aggravated by the spinning and baking processes.
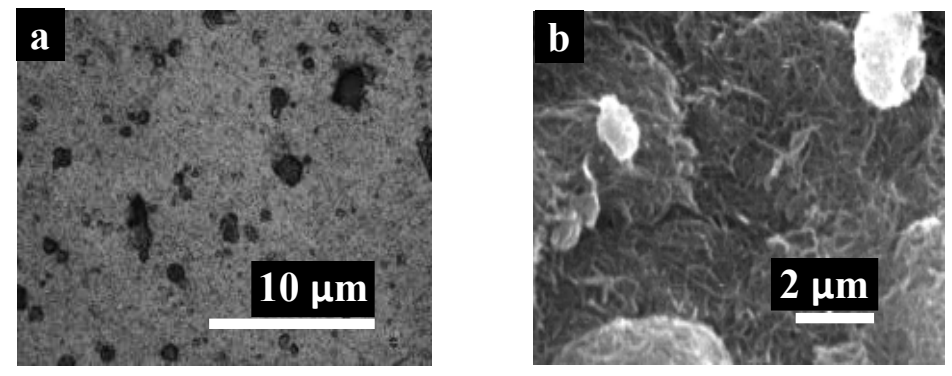

Figure 3. SEM images of five layers $(10 \mu \mathrm{m})$ of MWNT/PMMA indicating (a) the rough texture and (b) the clustering present in the samples.

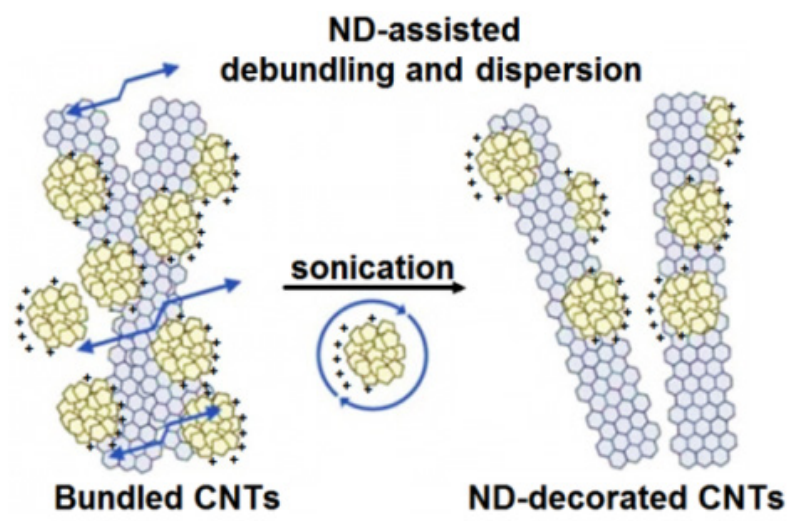

Figure 4. Dispersion mechanism of CNT bundles by charged DND clusters [18]. 
To reduce agglomeration and the sonication time, in the third and most successful approach, charged nanodiamond (ND) (zeta potential $+40 \mathrm{mV}$ ), a non-volatile dispersant, was mixed with MWNT (Nanostructured and Amorphous Materials, Inc. [17]) using dimethylsulfoxide (DMSO, Fisher Scientific), and PMMA [18]. The charged DND particles interact electrostatically with the MWCNTs and promote the formation of a shell of charged DNDs around a CNT core (figure 4). The charged DND-decorated CNTs repel each other, resulting in reduced agglomeration and better dispersion of the CNTs in the polymer solution. The dispersion of MWNTs assisted with DNDs and DMSO was remarkably better than that achieved by DMF (compare figures 4 and 5(a)). Specifically, the density of MWNT in the polymer matrix improved to $0.4 \mathrm{wt} . \%$ (40× improvement). Spinning the MWNT/DND/DMSO/PMMA mixture onto a substrate, even at very slow spin speeds $(<500 \mathrm{rpm})$, resulted in thin films with smooth surfaces (thickness as low as $300 \mathrm{~nm}$ and surface roughness on the order of $50 \mathrm{~nm}$ ) (figure 5 (b)). As shown in figures 5(c) and (d), the film morphology clearly indicates a very uniform network of MWNT and DND horizontally dispersed in the PMMA matrix. The presence of well-dispersed globular clusters of DND/PMMA coating the walls of the MWNT can also be clearly seen. These compositions exhibited the best film morphology as compared to the previous approaches.
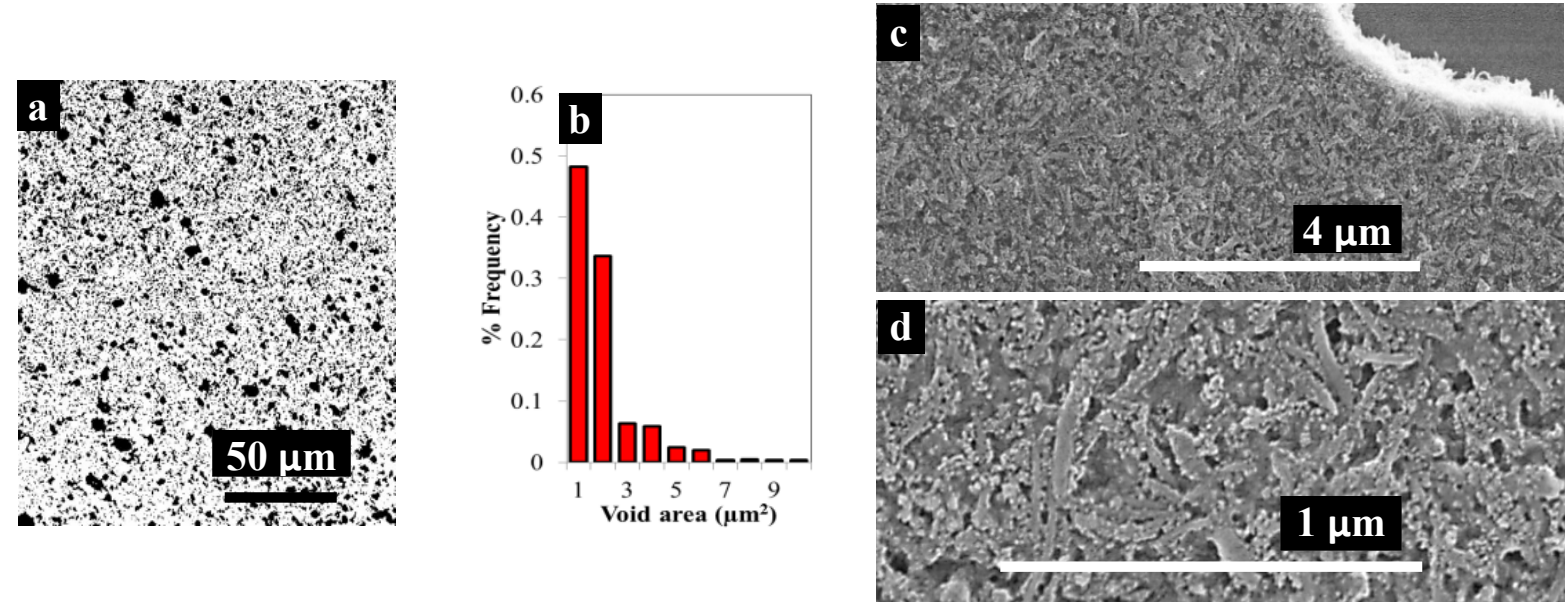

Figure 5. (a) Micro-scale morphology for the MWNT/DND/PMMA film. (b) Majority of the surface voids are less than $1 \mu \mathrm{m}^{2}$ in area and uniformly distributed across the sample. The surface roughness is $53 \mathrm{~nm}$. (c) SEM image of a $700 \mathrm{~nm}$ single layer MWNT/DND/PMMA showing a very uniform film with a porous mesh structure that can trap IR energy efficiently. The etched region in the top right corner is for measuring the film thickness. (d) Distributed clusters of DND decorated MWNT in a PMMA matrix can be clearly seen.

\section{Measurement results and discussion}

IR transmission measurements were carried out using a Perkin Elmer Spectrum GX in the spectral range of $714 \mathrm{~nm}$ to $5 \mu \mathrm{m}$ and normalized to the transmission of the glass substrate. A $150 \mathrm{~nm}$ thick silicon nitride layer, a commonly used absorber for microbolometers, was deposited on a glass substrate for comparison. For IR absorber films made from CNTs or carbon/graphite paints, the reflectance ranges from 0 to $5 \%$ at most for comparable spectral ranges [19]. Thus similar reflectance is expected for the samples in this work. Figures 6(a) and (b) present the IR transmission response of electrosprayed MWNT, spin-coated MWNT/PMMA 
and MWNT/DND PMMA nanocomposites, respectively. Figure 6(c) summarizes the IR absorbance as a function of the film thickness for the various nanocomposites. All approaches are able to achieve very high IR absorbance. All films, in contrast to silicon nitride, absorb IR in a broad spectral band and are not wavelength specific. This supports the hypothesis that the porous structure of the films aids in absorbing broadband IR energy. The MWNT/PMMA films (without DND) exhibit high absorbance and a flat spectral response. However, they are thick, non-uniform and rough, and as such unsuitable as thin-film IR absorbers. The electrosprayed MWNT films show excellent IR absorptivity but are hard to pattern. The MWNT/DND/PMMA films are not only stable but also exhibit high absorbance for film thicknesses in the range of 2 $\mu \mathrm{m}$ (figure 6(b)).
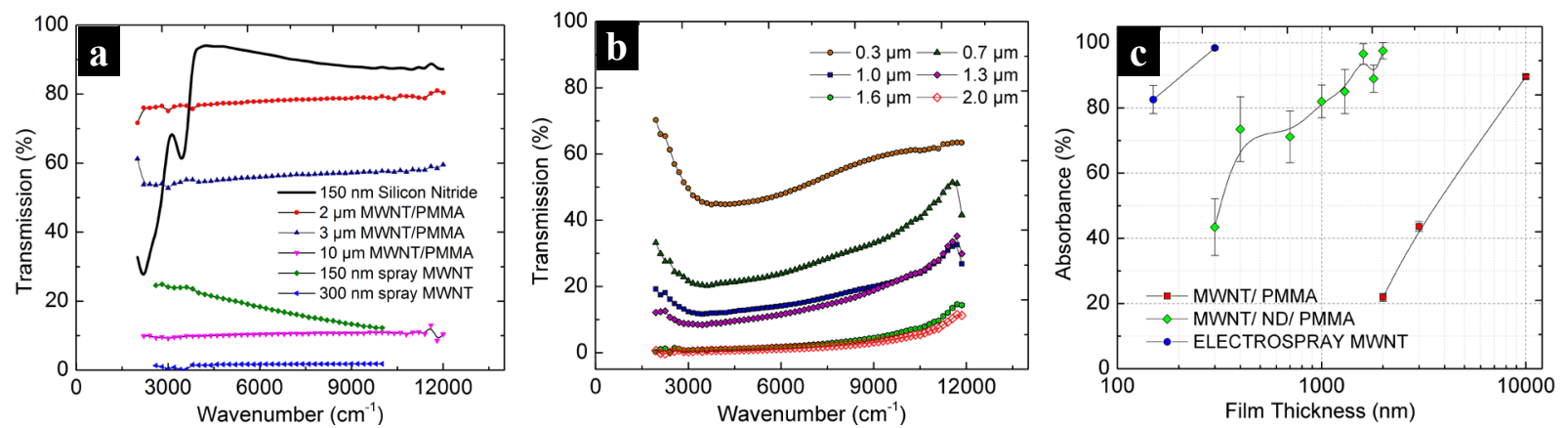

Figure 6. (a) IR transmission spectra for multilayer films of electrosprayed MWNT or spun MWNT/PMMA films compared with a $150 \mathrm{~nm}$ PECVD silicon nitride absorber. All spectra are normalized to the transmission of the glass substrate. The broadband nature of the MWNT films can be clearly seen. (b) IR transmission spectra for spin-coated MWNT/DND/PMMA films. (c) Trends for fractional IR absorbance as a function of film thickness for electrosprayed MWNT, MWNT/PMMA, and MWNT/DND/PMMA films.

To examine the temperature stability of the MWNT/DND films, the films were heated up to $300{ }^{\circ} \mathrm{C}$ and their IR characteristics were re-measured. The IR transmission of the $1.6 \mu \mathrm{m}$ thick film varies by less than $10 \%$ (figure 7 ), and there is no sample damage due to thermal cycling. Similar trends are seen for all other films, regardless of the deposition method.

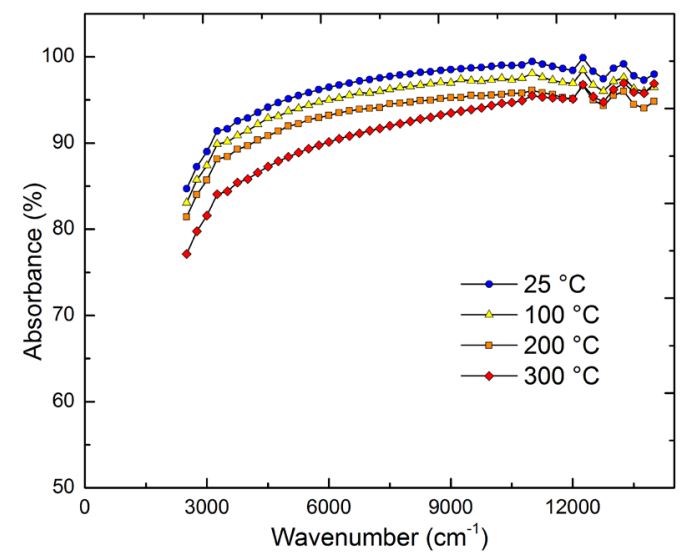

Figure 7. Effect of temperature on the absorption spectrum of a $1.6 \mu \mathrm{m}$ thick MWNT/DND/ PMMA film. The average deviation from nominal even at $300^{\circ} \mathrm{C}$ is less than $10 \%$. 


\section{CONCLUSIONS}

The fabrication of a CNT-based polymer nanocomposite was reported, which forms a thin film network that traps incident infrared radiation and absorbs it efficiently. Such thin films can be used as a lightweight absorber layers to increase the sensitivity of microbolometers and frequency shifting resonant IR detectors [20], [21] without adversely affecting their operation via mass loading. A broadband IR absorbance of $97 \%$ was obtained for a $1.6 \mu \mathrm{m}$ thick CNT-based film dispersed into PMMA using charged detonation nanodiamond particles. This film is mechanically and thermally stable, and electrically insulating. The spectral range of uniformly high absorbance is extended to the mid and far IR, since it is dependent on the structural properties of the film and not the material properties.

\section{ACKNOWLEDGMENTS}

This work was funded by ARL under contract W911NF and prepared through collaborative participation in the MAST CTA and by NSF under award \# 1002036.

\section{REFERENCES}

[1] A. Rogalski, Infrared Detectors, New York, Gordon and Breach, 2000.

[2] T. Bostrom, G. Westin and E. Wackelgard, Solar Energy Materials and Solar Cells, 91(1), 38 (2007).

[3] M. Ahn, Y.-H. Han, and S. Moon, Current Appl. Phys., 7(6), 617, (2007).

[4] W. Lang, K. Kühl and H. Sandmaier, Sensors and Actuators A, 34, 243, (1992).

[5] M. Gradhand and O. Breitenstein, Rev. Sci. Instrum. 76, 053702, (2005).

[6] J. Lehman, et al, Meas. Sci. and Tech., 14, 916, (2003).

[7] I. Mellouki, N. Bennaji, and N. Yacoubi, Infrared Phys. \& Tech., 50(1), 58, (2007).

[8] I. Mellouki, O. Touayar, T. Ktari, and N. Yacoubi, Infrared Phys. \& Tech., 45(4), 273, (2004).

[9] G. Biener, A. Niv, V. Kleiner, and E. Hasman, Optics Lett., 32, 994, (2007).

[10] J. Lehman, C. Engtrakul, T. Gennett, and A. Dillon, Appl. Optics, 44, 483, (2005).

[11] K. Mizuno, J. Ishii, H. Kishida, Y. Hayamizu, Satoshi Y, D. N. Futaba, M. Yumura, and K. Hata, Proc. Nat. Acad. Sci. USA, 16(15), 6044, (2009).

[12] www.nano-lab.com

[13] http://www.cheaptubesinc.com

[14] www.microchem.com

[15] http://www.fishersci.com

[16] E. T. Thostenson, Z. Ren, and T.-W. Chou, Composites Sci. and Tech., 61, 1899, (2001).

[17] www.nanoamor.com

[18] S. C. Hens, G. Cunningham, G. McGuire, and O. Shenderova, Nanosci. Nanotech. Lett., 3(1), (2011).

[19] J. Lehman, et al., Nano Lett., 9 3261(2010).

[20] V. J. Gokhale, S. Yu, and M. Rais-Zadeh, Proceedings of SPIE: Infrared Technology and Applications, (2012).

[21] V. J. Gokhale, J. Roberts, and M. Rais-Zadeh, Solid-State Sensors, Actuators and Microsystems Workshop, (2012). 